\title{
The aggregation rate constant of the discrete population balance model in hot melt fluidized bed coating process
}

\author{
Xin, W. ${ }^{\text {a }}$; Yan, Y. ${ }^{\text {b; }}$ Qing, X. ${ }^{\text {a,b* }}$; Long $\mathrm{W}^{\mathrm{a}, \mathrm{b}}$ \\ a International Joint Research Center of Low-Carbon Green Process Equipment; College of \\ Mechanical Engineering, Tianjin University of Science \&Technology, Tianjin 300222, China \\ b Tianjin Key Laboratory of Integrated Design and On-line Monitoring for Light Industry \& Food \\ Machinery and Equipment, Tianjin 300222, China.
}

*E-mail of the corresponding author: xuqing@tust.edu.cn

\begin{abstract}
During hot melt fluidized bed coating, particle agglomeration leads to nonuniform particle size. In this study, Population Balance Model (PBM) is used to establish the conservation of the size of particles in the system. In order to solve the population balance model, it is discretized. The aggregation kernel of the particles can be described by the Equi-partition of Kinetic Energy (EKE) kernel based on the gas dynamics theory. The EKE kernel is incorporated into a discrete population balance (DPB) model, and the effective aggregation rate constant is obtained by fitting with the experimental data.
\end{abstract}

Key words: Hot melt fluidized bed, PBM, DPB, EKE kernel, Aggregation rate constant. 


\section{Introduction}

Hot melt fluidized bed coating is an advanced technology for the preparation of microcapsules and it is commonly used in the food, pharmaceutical, and chemical industries. The coating process involves the agglomeration and breakage of the particles. In most studies of the model, the agglomeration model is used to describe the coating process. Tan et al (2004c) ${ }^{[1]}$ showed that the agglomeration rate of the particles was much greater than the breakage rate in the melt granulation experiments. For the coating process of particle groups, particles of different sizes are transformed into each other through nucleation, growth, and breakage. Through the introduction of the particle population balance model (PBM), a conservation relationship is established between particles of various sizes in the system. Anette et $\mathrm{al}^{[2]}$ established a theoretical model to quantitatively explain the agglomeration growth rate, which was in good agreement with the experiment; C.F.W. Sanders et $\mathrm{al}^{[3]}$ predicting high shearing granulation behavior in the granulation process based on discrete population balance (DPB) model; Tan et $\mathrm{al}^{[4,5]}$ deduced the EKE kernel based on particle growth kinetics theory to describe the net growth rate of hot melt spray fluid bed coating. In these models, the growth process of the particles is reflected from different aspects. However, the study on the constant of aggregation rate in the process of hot melt fluidized bed coating is rare.

This study only considers the agglomeration process in the coating process of hot melt fluidized bed. The main purpose is to incorporate the EKE kernel into a discrete population balance (DPB) model and use MATLAB for numerical simulation. The experimental data is fitted to a discrete population balance model to obtain an effective constant for aggregation rate.

\section{Simulation Methods}

\subsection{Establishment of a population balance model (PBM)}

In the process of hot melt fluidized bed coating, the agglomeration and breakage of particles resulted in non-uniform particle size. A population balance model (PBM) is introduced to establish a conservation relationship for each size particle in the system, describe the change of particle size distribution with time or space during the coating process, and reflect the process of particle growth and extinction. PBM is expressed by describing the rate of change of the particle size distribution density function. The model equation is: 


$$
\begin{aligned}
\frac{\partial n(t, v)}{\partial t} & =B^{0}(t, v)-\frac{\partial}{\partial v}[G(t, v) n(t, v)] \\
& +\frac{1}{2} \int_{0}^{v} \beta(t, v-\varepsilon, \varepsilon) n(t, v-\varepsilon) d \varepsilon-n(t, v) \int_{0}^{\infty} \beta(t, v \varepsilon) n(t, \varepsilon) d \varepsilon \\
& +\int_{0}^{\infty} S(t, \varepsilon) b(v, \varepsilon) n(t, \varepsilon) d \varepsilon-S(t, v) n(t, v) \\
& -O(t, v) n(t, v)
\end{aligned}
$$

where $n(t, v)$ is a number density function, $v$ and $\varepsilon$ are the mass of particles or granules, $B$ is the nucleation rate, $G$ is the growth rate, $\beta$ is an aggregation kernel, $S$ is a selection function, $b$ is a breakage function.

The aggregation kernel uses the EKE kernel described by Hounslow $(1998)^{[6]}$ based on the theory of gas dynamics. Expressed as:

$$
\beta_{i, j}=\beta_{0}(t)\left(l_{i}+l_{j}\right)^{2} \sqrt{\frac{1}{l_{i}^{3}}+\frac{1}{l_{j}^{3}}}
$$

where $\beta_{0}$ is the second-order rate constant, and $l_{i}$ and $l_{j}$ represent particles of size $\mathrm{i}$ and j. This model uses the particle flow dynamics theory described by Goldschmidt et $\mathrm{a}^{[7]}$ to provide a theoretical basis for the particle collision frequency in a fluidized bed. $\beta_{0}$ can be expressed as:

$$
\beta_{0}(t)=\psi g_{i j} \sqrt{\frac{3 \theta_{s}}{\rho}}
$$

where $\psi$ is defined as the ratio of the aggregation rate to the collision rate, $g_{i j}$ is the particle radial distribution function, $\rho$ is the particle density, $\theta_{s}$ is the particle temperature. Based on this work, it is considered that $\beta_{0}$ should be a constant for any set of experimental conditions, so in all the work here, $\beta_{0}$ can be described by the value of a single timeindependent parameter.

\subsection{Solution to the population balance equation}

In order to solve the equation of population balance model, this study uses the method of discretization ${ }^{[8]}$.It ensures correct prediction of particles number and volume for the aggregation terms. In the discretization scheme used, the length domain is divided into 
geometric intervals where the ratio of the upper and lower limits of each size interval is $r=\sqrt[3]{2}$. By assuming that the system is well-mixed, the discrete equation that gives the rate of change of the number of particles in the $i$ th interval is:

$$
\begin{aligned}
\frac{d N_{i}}{d t} & =N_{i-1} \sum_{j=1}^{i-2} 2^{j-i+1} \beta_{i-1, j} N_{j}+\frac{1}{2} \beta_{i-1, j-1} N^{2}{ }_{i-1} \\
& -N_{i} \sum_{j=1}^{i-1} 2^{j-1} \beta_{i, j} N_{j}-N_{i} \sum_{j=1}^{n_{e q}} \beta_{i, j} N_{j}
\end{aligned}
$$

$\mathrm{N}$ is the number of particles in a particular size class. Use MATLAB to solve the discretized numerical model.

\subsection{Calculation of the aggregation rate constant $\beta_{0}$}

The rate extraction problem is treated as a simple chi-square minimization process in the manner of Press et $\mathrm{al}^{[9]}$. In considering only the aggregation model, assuming that the aggregation rate is independent of time, the equation (2), (4) is simultaneously fitted with the mass-based experimental data to obtain the aggregated rate constant $\beta_{0}$ for the entire experiment. Using the integral fitting technique to minimize the chi-square method $\chi^{2}$ :

$$
\chi^{2}(p)=\sum_{i} \sum_{k}\left\{\left[\hat{W}_{i}\left(t_{k}\right)-\tilde{W}_{i}\left(t_{k}, p\right)\right]^{2}\right\}
$$

Where $\boldsymbol{P}$ is the fitting parameter vector, $\hat{W}_{i}$ is the mass-weighted particle size distribution of the experiment, and $\widetilde{W}_{i}$ is the predicted particle size distribution.

\section{Experimental}

Using the method of spray cooling granulation, the citric acid is subjected to granulation experiments using a fluidized spray granulation device. Experimental device flow chart shown in fig 3-1: 


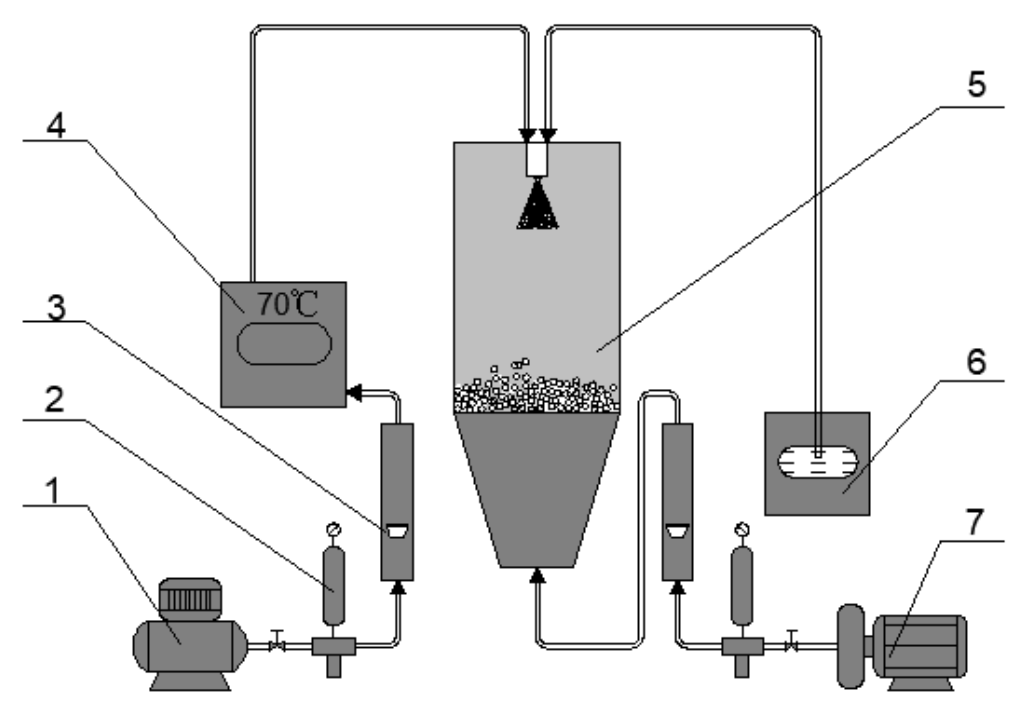

Fig 3-1 Citric acid experiment flow chart. 1, Air compressor; 2, Water separator; 3, Air flow meter; 4, Constant temperature water bath; 5, Spray fluidized bed; 6, Lipid; 7, Fan.

The experimental materials selected citric acid particles and lipid (butter), citric acid particles as the core material, and lipid as the wall material. $150 \mathrm{~g}$ of citric acid particles are weighed and frozen in a freezer at $-30^{\circ} \mathrm{C}$ for 24 hours. The lipid is heated and melted and sent to the nozzle, mixed with air in the nozzle, and then atomized into droplets. The citric acid particles are placed in a spray fluidized bed and the fluidized citric acid particles collide with the atomized droplets to wet, coat, and granulate.

The absolute pressure of atomization during the experiment is 0.6 MPa. The flow rate of citric acid particles is $0.5 \mathrm{~m} / \mathrm{s}$. The experiments are divided into 4 groups. The fluidization time is $0.5 \mathrm{~min}, 1.0 \mathrm{~min}, 1.5 \mathrm{~min}$ and $2.0 \mathrm{~min}$ respectively. Each set of experiments is repeated 3 times. At the end of the experiment, the fan is turned off and the nozzle spray is stopped. The experimental sample is taken out and sieved. The percentage of mass is used as an important criterion for evaluating granulation, and the particle size distribution of each set of experimental samples is determined.

\section{Results and discussion}

The particle size distribution of each experimental sample is shown in fig 4-1: 


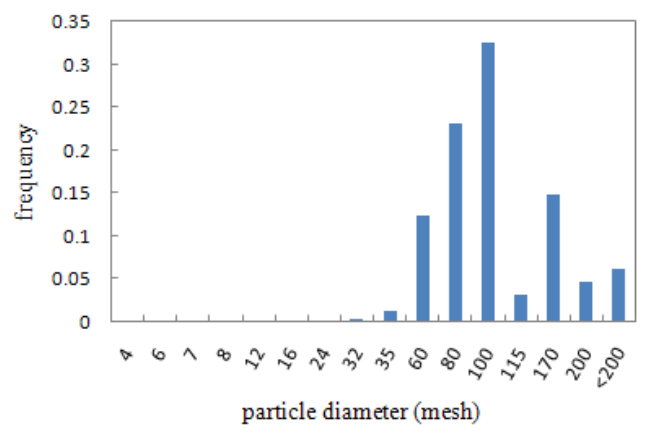

(a)t $=0 \mathrm{~min}$

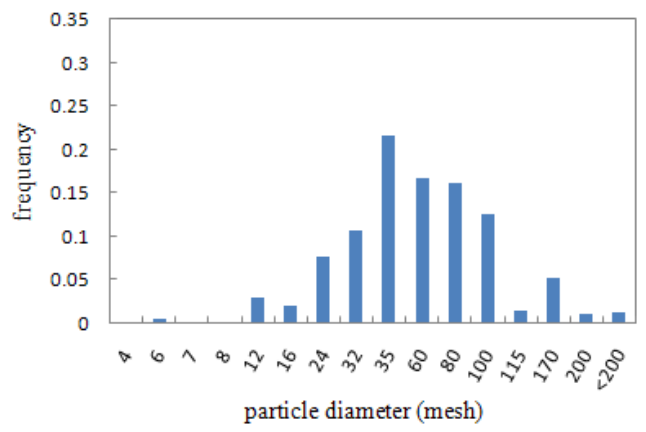

(c) $\mathrm{t}=1.0 \mathrm{~min}$

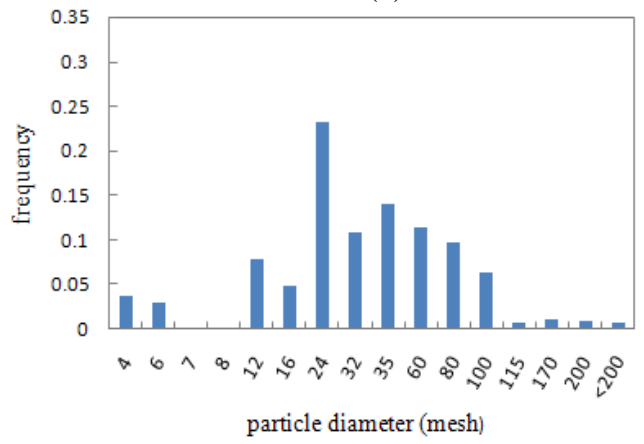

(e) $\mathrm{t}=2.0 \mathrm{~min}$

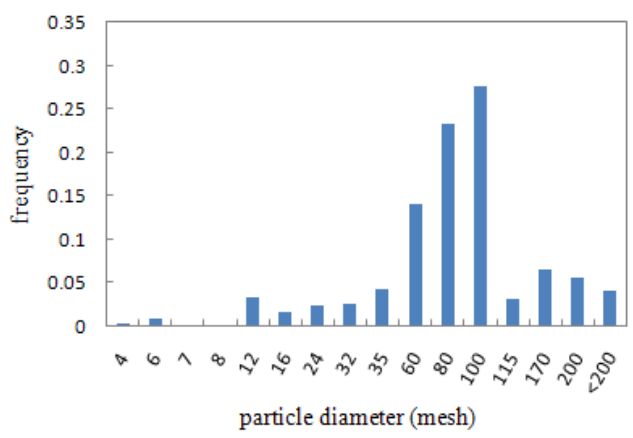

(b) $t=0.5 \mathrm{~min}$

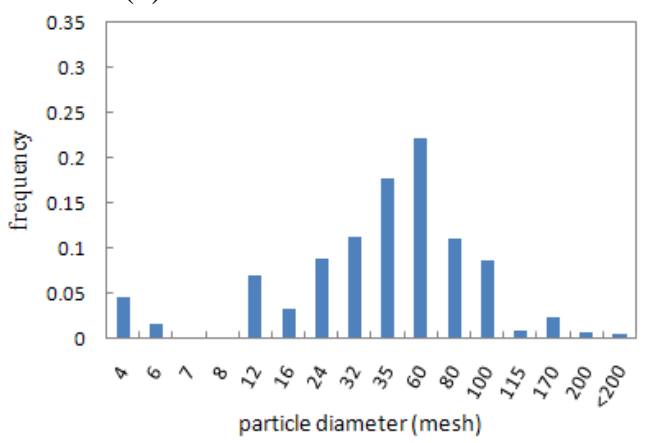

(d) $t=1.5 \mathrm{~min}$

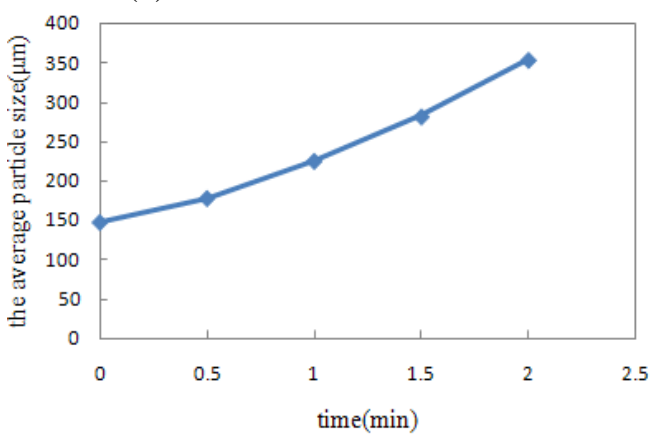

(f)Mean Size

Fig 4-1 Particle size distribution based on mass at different time.The last figure shows the change mean size.

As shown in fig. $4-1$, at $t=0 \mathrm{~min}$, that is, the citric acid particles are not embedding with lipid at the initial time. The distribution of particles is observed to be concentrated between 60 mesh and 200 mesh, with a particle size of 100 mesh particles having the highest mass percentage, accounting for $23 \%$. 
As shown in fig (b), the lipid is atomized and coated on citric acid particles. After spraying for $0.5 \mathrm{~min}$, the experiment is ended and the particle size distribution is analyzed. Compared with fig (a), the particle size distribution range is larger, mainly distributed between 12 and 200 mesh, with the largest distribution of citric acid particles between 60 mesh and 100 mesh. However, the percentage of particles with a particle size of 100 mesh is still the largest, accounting for $28 \%$. This is because the lipid is applied atomically to the fluidized citric acid particles so that the particles are coated and the diameter of the particles increases. However, when the coating is applied for $0.5 \mathrm{~min}$, the percentage of the particles between 4 and 6 mesh was almost zero.

In fig (c), the experiment is performed for $1.0 \mathrm{~min}$. It can be observed from the figure that the particle size distribution is mainly concentrated between 12 mesh and 100 mesh, among which the citric acid particles distributed between 24 mesh and 100 mesh are the most, and the citric acid particles with particle size 35 mesh are the most distributed, accounting for $21 \%$, Compared to fig (b), the percentage of particles in each particle size range increased. Particles between 115 mesh and 200 mesh gradually decreased with increasing experimental time. This is due to the fact that as the experimental time increases, more lipid and citric acid particles contact and collide, so that the particles are sufficiently agglomerated, the particles in a small size range are reduced, and the particle size is increased. Relative to fig (b), the particle size distribution of fig (c) is relatively uniform, showing a normal distribution as a whole.

Fig (d) shows that the entire experiment is 1.5 minutes. Compared to (c), large granules of citric acid are present, with an increase in granule size of 4 mesh citric acid particles, and few particles between 115 mesh and 200 mesh. The particle size range of the particles is also concentrated between 12 mesh and 100 mesh. At this point, the particle size distribution is 60 mesh with the largest percentage of particles, accounting for $22 \%$.

The particle size distribution in fig (e) is similar to that of fig (d). The particle size distribution range is basically the same and is mainly concentrated between 12 mesh and 100 mesh. However, the particle size distribution of 24 mesh particles is the largest, accounting for $23 \%$.

With the increase of the experimental time, citric acid is embedded in lipid, and the average particle diameter increases. This is because the lipid is sprayed onto the fluidized particles after atomization and the particles are coated.When the experiment time increases, the quality of the atomized wall material increases, while the quality of the core material does not change. Then, the mass-based particle size distribution of citric acid particles at different times is fitted to a discrete population balance model. Solve using MATLAB to get $\beta_{0}=7.5^{*} 10^{(-6)} \mathrm{kg} \mathrm{m}^{-1 / 2} \mathrm{~s}^{-1}$. 


\section{Conclusion}

A hot melt fluidized bed coating experiment using lipid-citric acid as the research object. The agglomeration of particles leads to non-uniform particle size, The particle size distribution range is larger, mainly concentrated between 12 mesh and 100 mesh, and the mass-based particle size distribution as a whole shows a normal distribution.

The experimental data is fitted to a discrete population balance model, and the aggregation rate constant $\beta_{0}=7.5 * 10^{(-6)} \mathrm{kg} \mathrm{m}^{-1 / 2} \mathrm{~s}^{-1}$.

The authors acknowledge Projects supported by the National Natural Science Foundation of China (Grant No.21506163 \& No. 31571906).

\section{References}

[1] Tan H.S.; Salman A.D.; Hounslow M.J. Kinetics of fluidised bed melt granulation IV: Selecting the breakage model. Powder Technology 2004, 143-144, 65-83.

[2] Anette P.; Klaus K., Bernhard C.L. Preparation of sustained release matrix pellets by melt agglomeration in the fluidized bed: Influence of formulation variables and modeling of agglomerate growth. European Journal of Pharmaceutics and Biopharmaceutics 2010, 74, 503-512.

[3] Sanders C.F.W.; Willemse A.W.; Salman A.D.; Hounslow W.J. Development of a predictive high-shear granulation model. Powder Technology 2003, 138, 18-24.

[4] Tan H.S.; Salman A.D.; Hounslow M.J. Kinetics of fluidized bed melt granulationII: Modelling the net rate of growth. Chemical Engineering Science 2006, 61, 39303941.

[5] Tan H.S.; Salman A.D.; Hounslow M.J. Kinetics of fluidised bed melt granulation V: Simultaneous modelling of aggregation and breakage. Chemical Engineering Science 2005, 60, $3847-3866$.

[6] Hounslow, M.J. The population balance as a tool for understanding particle rate processes. Kona Powder \& Particle Journal 2014, 16, 179-193.

[7] Goldschmidt, M.J.V. Hydrodynamic modelling of fluidised bed granulation. Ph.D. Thesis, Process Technology Institute of Twente, Twente University, Enschede, 2001.

[8] Hounslow, M.J.; Ryall, R.L.; Marshall, V.R. A discrete population balance model for nucleation, growth and aggregation. A.I.Ch.E. Journal 1988, 11(34), 1821-1832.

[9] Press,W.H.; Teukolsky, S.A.; Vetterling,W.T.; Flannery, B.P. Numerical Recipes in FORTRAN: The Art of Scientific Computing. seconded. Cambridge University Press, Cambridge, 1992. 